OPEN ACCESS

Edited by:

Hanzhang Lu,

Johns Hopkins University,

United States

Reviewed by:

Domenico Zaca,

Siemens, Italy

Baxter P. Rogers,

Vanderbilt University, United States

*Correspondence:

Ryan C. N. D'Arcy rdarcy@sfu.ca:

ryan_darcy@sfu.ca

Specialty section:

This article was submitted to

Brain Imaging Methods,

a section of the journal

Frontiers in Neuroscience

Received: 12 June 2019

Accepted: 09 September 2019

Published: 04 October 2019

Citation:

Grajauskas LA, Frizzell T, Song X and D'Arcy RCN (2019) White Matter fMRI Activation Cannot Be Treated as a Nuisance Regressor: Overcoming

a Historical Blind Spot.

Front. Neurosci. 13:1024

doi: 10.3389/fnins.2019.01024

\section{White Matter fMRI Activation Cannot Be Treated as a Nuisance Regressor: Overcoming a Historical Blind Spot}

\author{
Lukas A. Grajauskas ${ }^{1,2,3}$, Tory Frizzel/ ${ }^{3,4}$, Xiaowei Song ${ }^{1,3}$ and Ryan C. N. D'Arcy ${ }^{1,3,4,5 *}$ \\ ${ }^{1}$ Department of Biomedical Physiology and Kinesiology, Simon Fraser University, Burnaby, BC, Canada, ${ }^{2}$ Cumming School \\ of Medicine, University of Calgary, Calgary, AB, Canada, ${ }^{3}$ ImageTech Lab, Surrey Memorial Hospital, Fraser Health, Surrey, \\ BC, Canada, ${ }^{4}$ Faculty of Applied Sciences, Simon Fraser University, Burnaby, BC, Canada, ${ }^{5}$ Djavad Mowafaghian Centre \\ for Brain Health, University of British Columbia, Vancouver, BC, Canada
}

Despite past controversies, increasing evidence has led to acceptance that white matter activity is detectable using functional magnetic resonance imaging (fMRI). In spite of this, advanced analytic methods continue to be published that reinforce a historic bias against white matter activation by using it as a nuisance regressor. It is important that contemporary analyses overcome this blind spot in whole brain functional imaging, both to ensure that newly developed noise regression techniques are accurate, and to ensure that white matter, a vital and understudied part of the brain, is not ignored in functional neuroimaging studies.

Keywords: white matter, BOLD, fMRI, motion regression, fMRI analysis

\section{INTRODUCTION}

White matter is a vital part of the human brain, but in functional magnetic resonance imaging (fMRI), it remains largely overlooked and misunderstood. More than 15 years of evidence has shown that neural activation occurring in white matter can be detected using blood-oxygenation level dependent (BOLD) fMRI, and the existing literature has been well reviewed by Gawryluk et al. (2014b) and Gore et al. (2019). Despite this, white matter activation in fMRI is largely underrepresented and a great deal of research even ignores this evidence entirely and treats white matter in an inappropriate manner. Operating on the outdated assumption that white matter BOLD signal has no physiological component, many researchers have used the signal from white matter as a "nuisance regressor" to remove noise from fMRI signals. The evidence showing that physiological signals are present means the use of this technique is incorrect, however, these approaches continue to be employed (e.g., Jo et al., 2013; Power et al., 2014; Ciric et al., 2017). Several recent papers that used white matter as a nuisance regressor even cited literature supporting the existence of physiological signals in white matter, but then proceeded regardless of this evidence, offering little in the form of counterargument. For example, Barton et al. (2019) acknowledged the existence of fMRI detectable white matter activation, but then continued to use white matter signals as a nuisance regressor, with no justification or rationale. Similarly, Yang et al. (2019) acknowledged some of the white matter activation literature, but then also disregarded it, citing the "lack of neurons in white matter," a statement that is clearly anatomically incorrect (Kukley et al., 2007; García-Marín et al., 2010). These examples and others like them underscore the blind spot that has been carried into even the most contemporary fMRI research. The field needs to update its understanding and give appropriate credence to the evidence for detectable white matter BOLD signals in fMRI. 


\section{PHYSIOLOGICAL PRECEDENT}

The belief that white matter activation cannot be detected has become engrained in field of fMRI research, but this idea is not based on fundamental physiological principles. Instead, researchers have come to this belief simply because of a lack of reports in the literature. However, this absence may have simply stemmed from the difficulty of detecting the lower magnitude white matter BOLD signals on early $1.5 \mathrm{~T}$ scanners, as field strength has been shown to play a large role in the detection of white matter activity (Mazerolle et al., 2013; Gawryluk et al., 2014b). Despite this, there is physiological evidence that suggests a BOLD response can be detected in white matter, albeit one that is smaller and harder to detect. While our traditional understanding is that the BOLD response is primarily driven by the high energy demands of synaptic transmission, there is evidence that action potentials alone could drive a BOLD response. Histology indicates there is vasculature in white matter (Lierse and Horstmann, 1965; Duvernoy et al., 1981), and that this vasculature stems largely from the medullary artery which has no vascular interaction with cortical tissue (Nonaka et al., 2003; Akashi et al., 2017), meaning the energy demands of gray matter will not influence its oxygenation. The axons in white matter have been shown to be metabolically active (Takii et al., 2003), containing mitochondria all along their length (Misgeld et al., 2007), which require oxygen to produce ATP for cellular processes such as the re-establishment of ionic gradients after the transmission of action potentials. Oxygen uptake in isolated axons has been shown to be modified by changes in $\mathrm{Na}$ /K+ pump activity (Hargittai et al., 1987), and oxygen demand increases in axons undergoing repeated action potentials (Ritchie, 1967). Furthermore, a linkage between active axons and glia has been shown (Hargittai and Lieberman, 1991), where glia will activate potassium pumps to help restore ionic balance during an action potential (Petzold and Murthy, 2011) and therefore require oxygen in a task dependent manner. Overall, the evidence to support task dependent hemodynamic changes in white matter coupled with accumulating evidence from imaging should cause researchers to rethink their assumptions about white matter fMRI.

\section{REPORTS OF fMRI ACTIVATION IN WHITE MATTER}

Alongside this physiological precedent, more than 15 years of fMRI evidence has been published to support the existence of a detectable white matter BOLD signal, across a range of different tasks. One of the most robust methods for detection of white matter BOLD is using an "interhemispheric transfer task" to drive communication between hemispheres. This has consistently elicited detectible activation in the corpus callosum (Tettamanti et al., 2002; Omura et al., 2004; Weber et al., 2005; D'Arcy et al., 2006; Mazerolle et al., 2008; Gawryluk et al., 2009, 2011a). Mazerolle et al. (2010) further combined DTI with white matter BOLD to show that activated areas in the corpus callosum were structurally connected to the cortical areas activated by the interhemispheric transfer task. Fabri et al. (2011) also showed distinct patterns of activation in the corpus callosum for tactile, gustatory, visual, and motor tasks. Activation has also been shown in the corticospinal tract during swallowing (Mosier et al., 1999) and during a finger tapping task (Gawryluk et al., 2011b; Mazerolle et al., 2013), in the optic radiations during a visual task (Brandt et al., 2000), and in the internal capsule during a symbol digit modalities test (Gawryluk et al., 2014a). Weis et al. (2011) also found white matter activation during a memory task in both healthy controls and Alzheimer's patients. White matter activation has also been studied in resting state MRI, for example Ding et al. (2013) showed that functional connectivity within white matter tracts in the corpus callosum and optic radiations was greater within the tract than when compared with other voxels matched in distance from the seed. Ding et al. (2018) also used resting state MRI to show that there was connectivity between cortical regions and specific white matter tracts. This group was also able to create "functional connectivity tensors" similar to diffusion tensors by assessing the degree of signal correlation between a voxel and its adjacent neighbors, and representing the directionality of this correlation as a tensor (Ding et al., 2016). These functional correlation tensors present an interesting new methodology for investigating white matter activity and connectivity within the brain. Overall, these studies have taken great care to rule out partial volume effects (Gawryluk et al., 2014b), and the correspondence between the nature of the task and the white matter tract activated gives credence to the idea that this represents real activation, not simply artifact.

To add to this, recently published work has also made it clear that white matter has a different hemodynamic profile than the models that have been traditionally used in gray matter. A recent paper focused on characterizing the hemodynamic response function (HRF) at different depths of white matter was published in Nature Communications (Li et al., 2019), showing that the HRFs differed significantly from gray matter, and even varied within the white matter based on the depth of the tract. Importantly, the authors confirmed prior work by Courtemanche et al. (2018) which evaluated the differences between gray matter and white matter HRFs. The differences in HRFs between gray matter and white matter also may have contributed to the lack of reports of white matter BOLD activation, as analysis techniques using the traditional gray matter HRF would not effectively capture the hemodynamic profile of white matter, further perpetuating the blind spot surrounding white matter BOLD. A number of new MRI techniques are promising for the detection of white matter BOLD, but at the very least, researchers should make use of a white matter specific HFR.

\section{NEW TOOLS FOR BETTER DETECTION OF WHITE MATTER BOLD fMRI}

The increasing evidence and acceptance surrounding white matter BOLD fMRI is well timed, as a number of technological advances allow for the better detection of white matter activation. Increases in spatial resolution will allow for the isolation of signals coming from small white matter tracts, 
and increases in temporal resolution will allow for better modeling of white matter hemodynamic responses and better characterization of resting state correlations. The increasing accessibility of ultra-high field scanners (7T and above) will allow for investigations with high spatial resolution, as well as improved signal- and contrast- to-noise ratios, allowing for enhanced detection and characterization of white matter signals. Multiband excitation, a technique which optimizes tradeoffs related to spatial resolution in shorter time limits, is more available (Poser and Setsompop, 2018), and highdensity channel coils have also become more common, further increasing spatial and temporal resolution through parallel imaging (Hardy et al., 2008). Additionally, new developments in other neuroimaging modalities such as magnetoencephalography will allow for multi-modal investigations of white matter function (Papadelis et al., 2012; Yoshida et al., 2017). All of these techniques are already challenging previously established limits in functional neuroimaging (Feinberg and Setsompop, 2013; Petridou et al., 2013), and will have a particularly large impact on the characterization of white matter signals.

\section{DISCUSSION}

White matter represents a major component of functional neural tissue and plays a critical role in neural networks. Despite this, white matter activation has become a blind spot in fMRI research. Because it could not be easily detected in the early development stages of fMRI, an assumption developed that white matter activation did not exist or was not detectable. This assumption led to a self-fulfilling prophecy as analysis methods were designed to prioritize the detection of gray matter activation. Given the

\section{REFERENCES}

Akashi, T., Takahashi, S., Mugikura, S., Sato, S., Murata, T., Umetsu, A., et al. (2017). Ischemic white matter lesions associated with medullary arteries: classification of MRI findings based on the anatomic arterial distributions. Am. J. Roentgenol. 209, W160-W168. doi: 10.2214/AJR.16.17231

Bartoň, M., Mareček, R., Krajčovičová, L., Slavíček, T., Kašpárek, T., Zemánková, P., et al. (2019). Evaluation of different cerebrospinal fluid and white matter fMRI filtering strategies-quantifying noise removal and neural signal preservation. Hum. Brain Mapp. 40, 1114-1138. doi: 10.1002/hbm. 24433

Brandt, T., Stephan, T., Bense, S., Yousry, T. A., and Dieterich, M. (2000). Hemifield visual motion stimulation: an example of intrehemispheric crosstalk. NeuroReport 11, 2803-2809. doi: 10.1097/00001756-20000821000039

Ciric, R., Wolf, D. H., Power, J. D., Roalf, D. R., Baum, G. L., Ruparel, K., et al. (2017). Benchmarking of participant-level confound regression strategies for the control of motion artifact in studies of functional connectivity. Neuroimage 154, 174-187. doi: 10.1016/j.neuroimage.2017.03.020

Courtemanche, M. J., Sparrey, C. J., Song, X., MacKay, A., and D'Arcy, R. C. N. (2018). Detecting white matter activity using conventional 3 Tesla fMRI: an evaluation of standard field strength and hemodynamic response function. NeuroImage 169, 145-150. doi: 10.1016/j.neuroimage.2017.12.008

D’Arcy, R. C. N., Hamilton, A., Jarmasz, M., Sullivan, S., and Stroink, G. (2006). Exploratory data analysis reveals visuovisual interhemispheric transfer in functional magnetic resonance imaging. Magn. Reson. Med. 55, 952-958. doi: 10.1002/mrm.20839 state of the field and the clear evidence of white matter activation, these assumptions need to be revisited otherwise the researchers will continue to proceed with a blind spot that encompasses approximately $50 \%$ of the functional neural tissue in the brain. At a minimum, fMRI analysis methodology is best to restrict the choice of nuisance regressors to non-neural tissue, such as CSF. While this paper offers a caution, it also has a more exciting perspective. Accepting the existence of detectable white matter activation opens up a host of new research questions, such as altered white matter function in injury or disease states, direct measures of functional connectivity in neural networks, and neuroplasticity changes at the network level. With increasing temporal and spatial resolution in modern MRIs, expanding our research field to include white matter activation has become readily accessible. All it will take to open up an exciting new chapter in fMRI research is for scientists to move past this outdated blind spot, and begin answering new questions using white matter BOLD fMRI.

\section{AUTHOR CONTRIBUTIONS}

LG contributed as primary author. TF assisted in writing the manuscript. XS and RD'A assisted in editing the manuscript and acted as lab PI.

\section{FUNDING}

This study received support from the Natural Sciences and Engineering Research Council Discovery Grant \#206875.

Ding, Z., Huang, Y., Bailey, S. K., Gao, Y., Cutting, L. E., Rogers, B. P., et al. (2018). Detection of synchronous brain activity in white matter tracts at rest and under functional loading. Proc. Nat. Acad. Sci. U.S.A. 115, 595-600. doi: 10.1073/pnas.1711567115

Ding, Z., Newton, A. T., Xu, R., Anderson, A. W., Morgan, V. L., and Gore, J. C. (2013). Spatio-Temporal correlation tensors reveal functional structure in human brain. PLoS One 8:e82107. doi: 10.1371/journal.pone.0082107

Ding, Z., Xu, R., Bailey, S. K., Wu, T., Morgan, V. L., Cutting, L. E., et al. (2016). Visualizing functional pathways in the human brain using correlation tensors and magnetic resonance imaging. Magn. Reson. Imag. 34, 8-17. doi: 10.1016/j. mri.2015.10.003

Duvernoy, H. M., Delon, S., and Vannson, J. L. (1981). Cortical blood vessels of the human brain. Brain Res. Bull. 7, 519-579. doi: 10.1016/0361-9230(81)90007-1

Fabri, M., Polonara, G., Mascioli, G., Salvolini, U., and Manzoni, T. (2011). Topographical organization of human corpus callosum: an fMRI mapping study. Brain Res. 1370, 99-111. doi: 10.1016/j.brainres.2010.11.039

Feinberg, D. A., and Setsompop, K. (2013). Ultra-fast MRI of the human brain with simultaneous multi-slice imaging. J. Magn. Reson. 229, 90-100. doi: 10.1016/j. jmr.2013.02.002

García-Marín, V., Blazquez-Llorca, L., Rodriguez, J. R., Gonzalez-Soriano, J., and DeFelipe, J. (2010). Differential distribution of neurons in the gyral white matter of the human cerebral cortex. J. Comp. Neurol. 518, 4740-4759. doi: 10.1002/ cne. 22485

Gawryluk, J. R., Brewer, K. D., Beyea, S. D., and D'Arcy, R. C. N. (2009). Optimizing the detection of white matter fMRI using asymmetric spin echo spiral. NeuroImage 45, 83-88. doi: 10.1016/j.neuroimage.2008. 11.005 
Gawryluk, J. R., D’Arcy, R. C. N., Mazerolle, E. L., Brewer, K. D., and Beyea, S. D. (2011a). Functional mapping in the corpus callosum: a 4T fMRI study of white matter. NeuroImage 54, 10-15. doi: 10.1016/j.neuroimage.2010.07.028

Gawryluk, J. R., Mazerolle, E. L., Brewer, K. D., Beyea, S. D., and D’Arcy, R. C. N. (2011b). Investigation of fMRI activation in the internal capsule. BMC Neurosci. 12:56. doi: 10.1186/1471-2202-12-56

Gawryluk, J. R., Mazerolle, E. L., Beyea, S. D., and D 'Arcy, R. C. N. (2014a). Functional MRI activation in white matter during the symbol digit modalities test. Fron. Hum. Neurosci. 8:589. doi: 10.3389/fnhum.2014.00589

Gawryluk, J. R., Mazerolle, E. L., and D'Arcy, R. C. N. (2014b). Does functional MRI detect activation in white matter? a review of emerging evidence, issues, and future directions. Front. Neurosci. 8:239. doi: 10.3389/fnins.2014.00239

Gore, J. C., Li, M., Gao, Y., Wu, T., Schilling, K. G., Huang, Y., et al. (2019). Functional MRI and resting state connectivity in white matter - a mini- review. Magn. Reson. Imag. 63, 1-11. doi: 10.1016/j.mri.2019.07.017

Hardy, C. J., Giaquinto, R. O., Piel, J. E., Rohling, K. W., Marinelli, L., Blezek, D. J., et al. (2008). 128-channel body MRI with a flexible high-density receiver-coil array. J. Magn. Reson. Imag. 28, 1219-1225. doi: 10.1002/jmri.21463

Hargittai, , P. T., and Lieberman, E. M. (1991). Axon-glia interactions in the crayfish: glial cell oxygen consumption is tightly coupled to axon metabolism. Glia 4, 417-423. doi: 10.1002/glia.440040410

Hargittai, P. T., Ginty, D. D., and Lieberman, E. M. (1987). A pyrene fluorescence technique and microchamber for measurement of oxygen consumption of single isolated axons. Anal. Biochem. 163, 418-426. doi: 10.1016/0003-2697(87) 90243-0

Jo, H. J., Gotts, S. J., Reynolds, R. C., Bandettini, P. A., Martin, A., Cox, R. W., et al. (2013). Effective preprocessing procedures virtually eliminate distancedependent motion artifacts in resting state FMRI. J. Appl. Math. 2013:9. doi: 10.1155/2013/935154

Kukley, M., Capetillo-Zarate, E., and Dietrich, D. (2007). Vesicular glutamate release from axons in white matter. Nat. Neurosci. 10:311. doi: 10.1038/nn1850

Li, M., Newton, A. T., Anderson, A. W., Ding, Z., and Gore, J. C. (2019). Characterization of the hemodynamic response function in white matter tracts for event-related fMRI. Nat. Commun. 10, 1-11. doi: 10.1038/s41467-01909076-2

Lierse, W., and Horstmann, E. (1965). Quantitative anatomy of the cerebral vascular bed with especial emphasis on homogeneity and inhomogeneity in small parts of the gray and white matter. Acta Neurol. Scand. 41, 15-19. doi: 10.1111/j.1600-0404.1965.tb01946.x

Mazerolle, E. L., Beyea, S. D., Gawryluk, J. R., Brewer, K. D., Bowen, C. V., and D'Arcy, R. C. N. (2010). Confirming white matter fMRI activation in the corpus callosum: co-localization with DTI tractography. NeuroImage 50, 616-621. doi: 10.1016/j.neuroimage.2009.12.102

Mazerolle, E. L., D’Arcy, R. C., and Beyea, S. D. (2008). Detecting functional magnetic resonance imaging activation in white matter: interhemispheric transfer across the corpus callosum. BMC Neurosci. 9:84. doi: 10.1186/14712202-9-84

Mazerolle, E. L., Gawryluk, J. R., Dillen, K. N. H., Patterson, S. A., Feindel, K. W., Beyea, S. D., et al. (2013). Sensitivity to white matter fMRI activation increases with field strength. PLoS One 8:e58130. doi: 10.1371/journal.pone.0058130

Misgeld, T., Kerschensteiner, M., Bareyre, F. M., Burgess, R. W., and Lichtman, J. W. (2007). Imaging axonal transport of mitochondria in vivo. Nat. Methods 4, 559-561. doi: 10.1038/nmeth1055

Mosier, K. M., Liu, W. C., Maldjian, J. A., Shah, R., and Modi, B. (1999). Lateralization of cortical function in swallowing: a functional MR imaging study. Am. J. Neuroradiol. 20, 1520-1526.

Nonaka, H., Akima, M., Hatori, T., Nagayama, T., Zhang, Z., and Ihara, F. (2003). Microvasculature of the human cerebral white matter: arteries of the deep white matter. Neuropathology 23, 111-118. doi: 10.1046/j.1440-1789.2003. 00486.x

Omura, K., Tsukamoto, T., Kotani, Y., Ohgami, Y., Minami, M., and Inoue, Y. (2004). Different mechanisms involved in interhemispheric transfer of visuomotor information. Neuroreport 15, 2707-2711.

Papadelis, C., Leonardelli, E., Staudt, M., and Braun, C. (2012). Can magnetoencephalography track the afferent information flow along white matter thalamo-cortical fibers? Neuroimage 60, 1092-1105. doi: 10.1016/j.neuroimage.2012.01.054

Petridou, N., Italiaander, M., van de Bank, B. L., Siero, J. C. W., Luijten, P. R., and Klomp, D. W. J. (2013). Pushing the limits of high-resolution functional MRI using a simple high-density multi-element coil design. NMR Biomed. 26, 65-73. doi: $10.1002 / \mathrm{nbm} .2820$

Petzold, G. C., and Murthy, V. N. (2011). Role of astrocytes in neurovascular coupling. Neuron 71, 782-797. doi: 10.1016/j.neuron.2011.08.009

Poser, B. A., and Setsompop, K. (2018). Pulse sequences and parallel imaging for high spatiotemporal resolution MRI at ultra-high field. NeuroImage 168, 101-118. doi: 10.1016/j.neuroimage.2017.04.006

Power, J. D., Mitra, A., Laumann, T. O., Snyder, A. Z., Schlaggar, B. L., and Petersen, S. E. (2014). Methods to detect, characterize, and remove motion artifact in resting state fMRI. Neuroimage 84, 320-341. doi: 10.1016/j.neuroimage.2013. 08.048

Ritchie, J. M. (1967). The oxygen consumption of mammalian non-myelinated nerve fibres at rest and during activity. J. Physiol. 188, 309-329. doi: 10.1113/ jphysiol.1967.sp008141

Takii, Y., Takoh, K., Nishizawa, M., and Matsue, T. (2003). Characterization of local respiratory activity of PC12 neuronal cell by scanning electrochemical microscopy. Electrochim. Acta 48, 3381-3385. doi: 10.1016/S0013-4686(03) 00408-0

Tettamanti, M., Paulesu, E., Scifo, P., Maravita, A., Fazio, F., Perani, D., et al. (2002). Interhemispheric transmission of visuomotor information in humans: fMRI evidence. J. Neurophysiol. 88, 1051-1058. doi: 10.1152/jn.2002.88.2. 1051

Weber, B., Treyer, V., Oberholzer, N., Jaermann, T., Boesiger, P., Brugger, P., et al. (2005). Attention and interhemispheric transfer: a behavioral and fMRI study. J. Cogn. Neurosci. 17, 113-123. doi: 10.1162/0898929052880002

Weis, S., Leube, D., Erb, M., Heun, R., Grodd, W., and Kircher, T. (2011). Functional neuroanatomy of sustained memory encoding performance in healthy aging and in Alzheimer's disease. Int. J. Neurosci. 121, 384-392. doi: 10.3109/00207454.2011.565892

Yang, Z., Zhuang, X., Sreenivasan, K., and Mishra, V. (2019). Robust motion regression of resting-state data using a convolutional neural network model. Front. Neurosci. 13:169. doi: 10.3389/fnins.2019.00169

Yoshida, F., Hirata, M., Onodera, A., Goto, T., Sugata, H., and Yorifuji, S. (2017). Noninvasive spatiotemporal imaging of neural transmission in the subcortical visual pathway. Sci. Reports 7:4424. doi: 10.1038/s41598-01704700-x

Conflict of Interest: The authors declare that the research was conducted in the absence of any commercial or financial relationships that could be construed as a potential conflict of interest.

Copyright (c) 2019 Grajauskas, Frizzell, Song and D'Arcy. This is an open-access article distributed under the terms of the Creative Commons Attribution License (CC BY). The use, distribution or reproduction in other forums is permitted, provided the original author(s) and the copyright owner(s) are credited and that the original publication in this journal is cited, in accordance with accepted academic practice. No use, distribution or reproduction is permitted which does not comply with these terms. 